\title{
Endoplasmic reticulum-mitochondria tethering in neurodegenerative diseases
}

\author{
Yi Liu and Xiongwei Zhu* (B)
}

\begin{abstract}
Endoplasmic reticulum (ER) and mitochondria are tubular organelles with a characteristic "network structure" that facilitates the formation of inter-organellar connections. As a result, mitochondria-associated ER membranes (MAMs), a subdomain of the ER that is tightly linked to and communicates with mitochondria, serve multiple physiological functions including lipid synthesis and exchange, calcium signaling, bioenergetics, and apoptosis. Importantly, emerging evidence suggests that the abnormality and dysfunction of MAMs have been involved in various neurodegenerative disorders including Alzheimer's disease, amyotrophic lateral sclerosis, and Parkinson's disease. This review will focus on the architecture and function of MAMs and its involvement in the neurodegenerative diseases.
\end{abstract}

Keywords: Mitochondria-associated ER membrane, Mitochondria-ER tethering, Alzheimer's disease, Parkinson's disease, Amyotrophic lateral sclerosis

\section{Background}

In 1990, Vance [1] found that crude rat liver mitochondrial fraction was capable of rapid and linked synthesis of phospholipids and also contained phosphatidylserine synthase (PSS) and phosphatidylethanolamine methyltransferase activities which were absent from highly purified mitochondria. This could be attributed to the presence of a membrane fraction ' $\mathrm{X}$ ' in the crude but not highly purified mitochondria. It turns out that this fraction ' $\mathrm{X}$ ', now commonly regarded as the mitochondrial associated membrane (MAMs), is a specialized subdomain of the endoplasmic reticulum (ER) with specific lipid and protein composition that is involved in the crosstalk with mitochondria. Phospholipid synthesis and exchange was the first identified function of MAMs. Later, it was demonstrated by electron tomography that ER and mitochondria are linked by high electron-dense tethers $(10 \mathrm{~nm}$ at the smooth ER and $25 \mathrm{~nm}$ at the rough ER) termed as mitochondria-ER tethers (a.k.a., Mitochondria-ER contact/crosslink/crosstalk/communication or MERC) [2, 3]. In fact, approximately $5 \sim 20 \%$ of the mitochondrial surface is juxtaposed $(10-30 \mathrm{~nm}$ distances) to specialized regions of the ER tubules [4-7]. Such a short distance suggests that the two organelles

\footnotetext{
* Correspondence: xiongwei.zhu@case.edu

Department of Pathology, Case Western Reserve University, Cleveland, $\mathrm{OH}, \mathrm{USA}$
}

(c) The Author(s). 2017 Open Access This article is distributed under the terms of the Creative Commons Attribution 4.0 International License (http://creativecommons.org/licenses/by/4.0/), which permits unrestricted use, distribution, and reproduction in any medium, provided you give appropriate credit to the original author(s) and the source, provide a link to the Creative Commons license, and indicate if changes were made. The Creative Commons Public Domain Dedication waiver (http://creativecommons.org/publicdomain/zero/1.0/) applies to the data made available in this article, unless otherwise stated. are tethered together by proteins on the juxtaposed membranes [7]. MERC appear to be stable structures. Although the ER and mitochondrial membranes form multiple and specific crosslink sites, it must be emphasized that they do not fuse but instead still maintain the organelles' distinct structures and characteristics which is crucial for them to serve as a major platform to carry out important physiological roles in the regulation of intracellular calcium homeostasis [6, 8-12], lipid metabolism [1, 13], mitochondrial fission [14], autophagosome formation [15] and apoptosis progression [16, 17]. In this review, we will focus on recent data addressing the structural composition and function of the MAMs in mammalian system and its potential involvement in various neurodegenerative diseases.

\section{ER-mitochondria tethering protein complexes}

The molecular details of ER-mitochondria tethers are largely unknown. Electron microscopic studies showed that the cleft of a MERC is typically dotted by electrondense areas that are widely accepted to be formed by protein complexes. The MAM fraction can be detached from mitochondria through the limited digestion with trypsin or Proteinase $\mathrm{K}$ which is suggestive of proteinaceous characteristics [7]. Indeed, several pairs of integral membrane proteins located on mitochondria and ER important for MERC formation and physical 
tethering of the organelles were identified (Fig. 1) [5]. Additional proteins that localize in the cleft of the MERC and participate in its biochemical activity or functional regulation might also, directly or indirectly, participate in MERC formation or maintenance such as by spacing apart the opposing membranes of the ER and the mitochondrion at a distance that allows the formation of MERC tethers.

\section{Mfn2 Tether}

Mitofusin 2 (Mfn2) is a large GTPase involved in the fusion of mitochondrial outer membrane. After the initial report from Luca Scorrano's group suggesting the potential involvement of Mfn2 in ER-mitochondrial tethering, Mfn2 is among the most widely studied ER-mitochondria tethering protein factors but its exact role in MERC regulation is still in hot debate. In 2008, Luca Scoranno and colleagues found that Mfn2 ablation caused dramatic defects in ER morphology in vitro in mouse embryonic fibroblasts(MEFs) and HeLa cells [18]. It turned out that Mfn2 is enriched at MERC and is required for the juxtaposition of ER to mitochondria [18]. ER-located Mfn2 interacts in trans with mitochondrial mitofusins (i.e., Mfn1 or Mfn2) to form trans-organelle hetero- or homo- dimer tethers to bridge the mitochondria onto ER which allows efficient calcium transfer between ER and mitochondria $[18,19]$. The confocal microscopy analysis revealed that the distance between ER and mitochondria increases in cells lacking Mfn2 which impairs mitochondrial calcium uptake $[18,19]$. Indeed, the presence and enrichment of Mfn2 in the interface between ER and mitochondria is directly demonstrated by immunoelectron microscopy in Melanocytes [20]. Moreover, Mfn2-mediated ER-mitochondria tethering is regulated by MitoL-dependent activation of mitochondrial Mfn2 through ubiquitination and MitoL ablation inhibited Mfn2 complex formation and caused Mfn2 mislocalization from MAM to non-MAM ER [21]. Owing to its well-known role in the tethering of adjacent membranes, Mfn2 is widely accepted as a major regulator of MERC in different tissues

$[18,19,22]$. However, such a view was challenged by a quantitative electron microscopic study followed by a series morphological and functional studies from multiple groups: In 2012, Pierre C et al. reported that the percentage of mitochondria in close contact with ER tubules was increased in Mfn2 defective MEFs when compared to wildtype MEFs (4.91\% vs $2.25 \%$,

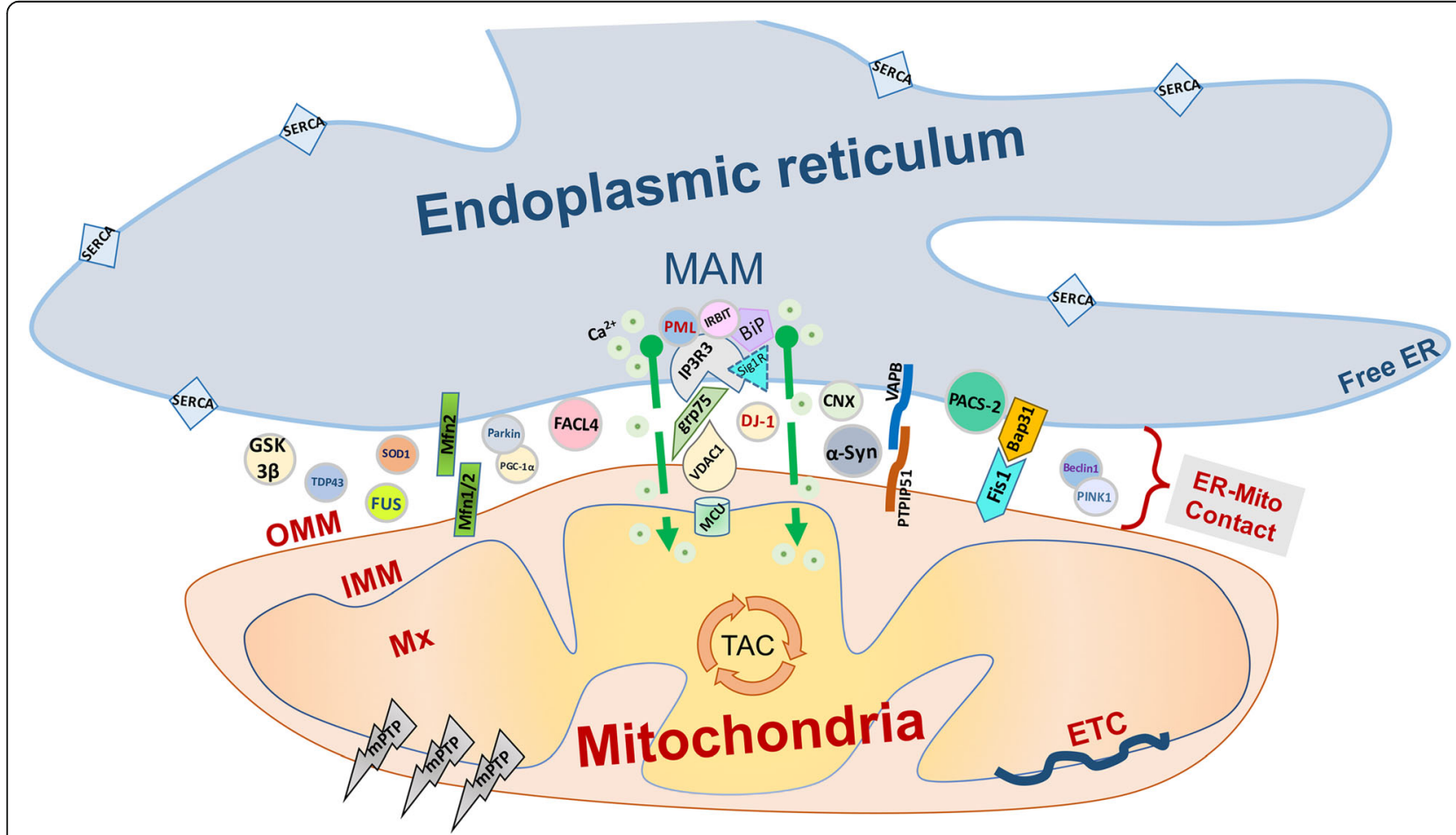

Fig. 1 Global view of the architecture/choreography of ER-mitochondria contacts. As depicted, a part of ER tubule and mitochondria form quasi-synaptic structure. Several pairs of integral membrane proteins located on mitochondria and ER important for MERC formation and physical tethering of the organelles were identified, including Mfn1/2 tether, Fis1-Bap31 tether, VAPB-PTPIP51 tether and IP3R-grp75-VDAC1 tripartite complex. The latter is essential for the efficient $\mathrm{Ca}^{2+}$ transfer from the ER to mitochondria. MAM: mitochondria associated ER membrane, OMM = outer mitochondrial membrane, IMM: inner mitochondrial membrane, Mx = matrix, ETC: electron transport chain, TAC: tricarboxylic acid cycle 
the distance between ER and mitochondria was restricted to $<20 \mathrm{~nm}$ in the study). In 2015, Riccardo Filadia et al. confirmed this EM findings that the ER-mitochondria crosslink was increased in Mfn2 defective MEFs and SH-SY5Y cells and provided functional evidence of an elevated calcium flow from ER to mitochondria in Mfn2 defective MEFs [23]. While they also confirmed the confocal microscopy results from Scoranno's group that Mfn2 deficiency caused a net decrease in the overlapping area between ER and mitochondria, they noted that the dramatic changes in mitochondrial morphology caused by Mfn2 ablation resulted in changes in mitochondrial area which could make classical colocalization analysis unsuitable. Excluding the confounding effects of area change, they found that mitochondrial perimeter colocalizing with the ER actually increased in Mfn2 deficient cells [23]. Other groups also reported that Mfn2 knockdown increased ER-mitochondria tethering and calcium transfer from ER to mitochondria [24]. Along this line, deficiency in Gp78 (a.k.a. autocrine motility factor receptor, AMFR), an endoplasmic-reticulum (ER)-associated protein degradation E3 ubiquitin ligase involved in the degradation of Mfn2, caused significantly decreased rough ER-mitochondria crosstalk as evaluated by EM which could be blocked by Mfn2 knockdown, suggesting that Gp78 might promote ER-mitochondria interaction through degradation of Mfn2 [25]. Collectively, these studies strongly challenges the role of Mfn2 as an essential component of ER-mitochondrial tethering but argues for a role of $\mathrm{Mfn} 2$ as a negative regulator of organelle apposition. Most recently, Luca Scorrano's group responded to these challenges by providing further ultramorphometric and confocal microscopic evidence based on unbiased fluorescent probes of ER-mitochondrial proximity to demonstrate that Mfn2 ablation increases ER-mitochondria distance which resulted in impaired mitochondrial calcium uptake in an mitochondrial calcium uniporter (MCU)-independent manner in MEF cells [22], yet the number of ER-mitochondria contacts were not directly addressed in this study and it is not clear whether and how larger ER-mitochondrial distance may affect ER-mitochondrial tethering. Further research is needed to fully elucidate the role of Mfn2 at MERC.

\section{VAPB-PTPIP51 Tether}

Vesicle-associated membrane protein-associated protein $\mathrm{B}$ (VAPB) is an integral protein in the ER membrane involved in ER unfolded protein response and the regulation of cellular calcium homeostasis [26]. Most recently, it was demonstrated that VAPB interacts with mitochondrial outer membrane protein tyrosine phosphatase-interacting protein-51 (PTPIP51) [27]. Overexpression of either proteins increase, while knockdown of either protein decreases, ER-mitochondria tethering along with functional changes such as calcium exchange between the two organelles, suggesting that this pair of proteins forms another molecular scaffold to tether the two organelles [28].

\section{Fis1-Bap31 Tether}

As an ER protein-sorting factor, B-cell receptor-associated protein 31 (Bap31), an abundant $28-\mathrm{kDa}$ integral membrane chaperone protein of the ER, forms several large protein complexes and controls the fate of newly synthesized integral membrane proteins. An earlier study demonstrated that outer membrane associated active caspase 8 could cleave ER-associated Bap31 and the cleavage product p20Bap31 mediated mitochondrion-ER cross talk through a calcium-dependent mechanism [29]. The overexpression of P20Bap31 could lead to early release of calcium from ER and concomitant uptake of calcium into mitochondria [30], implicating the functional involvement of Bap31 in ER-mitochondrial tethering. More recently, it was demonstrated that mitochondrial fission protein Fission 1 homologue (Fis1) interacts with Bap31 and forms an ER-mitochondrial platform which is essential in the recruitment and activation of procaspase 8 and the conveyance of the apoptotic signal from mitochondria to ER [31]. Given that Fis1-Bap31 interaction is also present in normal, non-apoptotic cells [31], it likely constitutes a preformed scaffold complex to tether ER-mitochondria together that may have roles broader than apoptotic signaling. The role of Bap31 in ER-mitochondria tethering may be modulated by phosphofurin acidic cluster sorting protein 2 (PACS-2), a multifunctional cytosolic sorting protein: depletion of PACS-2 caused Bap-31-dependent mitochondrial fragmentation and uncoupling from the ER along with inhibition of calcium signal transmission [32]. More recently, it was demonstrated that mammalian target of rapamycin complex 2 regulates the integrity of MAM by Akt-dependent phosphorylation of PACS-2 [33] although how PACS-2 promotes MAM integrity and regulates its composition is not entirely clear.

\section{IP3R3-Grp75-VDAC1}

The voltage-dependent anion channel (VDAC) of the outer mitochondrial membrane interacts with the ER calcium-release channel inositol 1,4,5-trisphosphate receptor (IP3R) through the molecular chaperone glucose-regulated protein 75 (grp75), which is essential for the efficient calcium transfer from the ER to mitochondria [34, 35]. 
Despite such an important functional role, this tripartite complex may not have a tethering role, but rather a MERC spacing/filling function that derives from functionally coupling ER and mitochondria in calcium exchanges. Nevertheless, the ER-resident protein Sigma1R (Sig-1R) stabilizes MAM by interacting with VDAC and IP3R and prolongs the calcium signaling in MAMs [36].

\section{Functions of MAM}

Mitochondria and ER have crucial but distinctive roles in mammalian cells. ER-mitochondria tethering provides a platform facilitating the crosstalk between these two organelles which appears essential for the function of these organelles. Indeed, it becomes increasingly clear that MAM plays an important role in various cellular processes critical for cell survival and death, which is briefly discussed below.

\section{Phospholipid Synthesis and Exchange}

While lipid synthesis largely occurs in the ER, it needs the assistance of other organelles since several of the key enzymes are located on the membrane of other organelles such as mitochondria [13, 37]. MAMs were initially described as an ER subdomain enriched in proteins involved in lipid metabolism such as PSS1 and PSS2 with an ascribed function as a platform for lipid biosynthesis and exchange $[1,38]$. Indeed, phosphatidylserine is first synthesized in the MAM by PSS1 and PSS2 [39]; then transferred to the closely apposed mitochondrion, where a decarboxylase in the inner mitochondrial membrane converts it to phosphatidylethanolamine; to make phosphotidylcholine, phosphatidylethanolamine is transferred back to the ER, where a methyltransferase converts it into phosphatidylcholine, a major component of the cell membrane. Phosphotidylcholine must be transferred back to mitochondria again since it is present in mitochondrial membrane [13]. Later studies revealed that MAMs are also the site of triacylglycerol synthesis and steroidogenesis [40]. Another lipid metabolism associated enzyme enriched in MAMs is Long-chain-fatty-acid-CoA ligase 4 (FACL4), also known as acyl-CoA synthetase long-chain family member 4 , which mediates the ligation of fatty acids to coenzyme $\mathrm{A}(\mathrm{CoA})$ and other cholesterol metabolites. Acyl-coenzyme A:cholesterol acyltransferase-1 that catalyzes the formation of cholesterol esters and diacylglycerol acyltransferase are also enriched in MAMs [41, 42].

\section{Calcium Transfer}

The calcium homeostasis of mitochondria is of vital significance to mitochondria and cell as a whole. On one hand, moderate loading of mitochondrial calcium has an important physiological function to stimulate ATP production through calcium-dependent activation of the key metabolic enzymes in the Krebs cycle such as pyruvate dehydrogenase and $\alpha$-ketoglutarate dehydrogenase [43]. On the other hand, calcium overloading of mitochondria causes the opening of mitochondrial permeability transition pore (mPTP) and leads to cell apoptosis [44]. ER is the intracellular calcium store and MAMs facilitate the calcium transfer between ER and mitochondria [9, 11]. While outer mitochondrial membrane is permeable to calcium through VDAC1, the inner mitochondrial membrane is not and calcium needs to go through MCU in the inner mitochondrial membrane [45-47]. As a low affinity calcium channel, MCU requires high calcium levels which can be efficiently achieved by the MAM through IP3R3-grp75-VDAC1 complex: the calcium released from ER through IP3R to the VDAC1 on the OMM leads to localized sites of calcium influx in the intermembrane space and creates the microdomain of high $\left[\mathrm{Ca}^{2+}\right]$ (calcium puff) close to MCU and thus facilitate the calcium uptake by MCU [48, 49]. The IP3R3-grp75-VDAC1 complex also serves as a molecular scaffold for other calcium handling players (Sig-1R [36], BiP, Bcl-2 [50], IRBIT [51], etc.), which may be essential for the fine-tuning of the calcium signaling of IP3R3-grp75-VDAC1 axis at the MAM.

\section{Regulation of Mitochondrial Fission}

Mitochondria are highly dynamic organelles that undergo continuous fusion and division which is critical for the homeostasis of mitochondria [52]. Mitochondrial fission involves the translocation of cytosolic dynamin-like protein (DLP1) to the outer membrane of mitochondria where it oligomerizes into ring-like structures that wrap around the mitochondrial constriction sites [14, 53, 54]. Interestingly, during fission, mitochondria appeared to be constricted at the point of contact with the ER. In both yeast and mammalian cells, Dnm1 (DLP1 yeast ortholog)/DLP1 localizes to the mitochondrial membrane constriction site wrapped by ER tubules [14]. Three-dimensional electron microscopic study of the structure of MERC in Saccharomyces cerevisiae revealed that mitochondria-associated ER tubules might mediate the formation of mitochondrial constriction sites [14]. The presence of ER tubules at mitochondrial constriction and fission sites has been further evidenced using a two-color STORM super-resolution study [55]. In fact, the perimeter of mitochondria tubules circumscribed by ER decreased by 30\% when compared with the uncircumscribed which better fit for the action of narrower DLP1 ring [14]. A later 
study revealed that the ER-localized protein inverted formin 2 is activated during fission to polymerize actin, which in turn might generate the driving force for initial mitochondrial constriction at the ER-mitochondria contact site [56].

\section{Apoptosis}

Both mitochondria and ER play a role in apoptosis and MERC provides a platform for the two organelles to exchange signals to regulate and coordinate a rapid and orderly cell demise [57]. The Fis1-Bap31 tethering complex (as discussed in "Fis1-Bap31 tether" earlier) allows for apoptotic signals being transferred from mitochondria to the ER by recruiting and activating caspase 8 which cleaves Bap31 into the pro-apoptotic p20Bap31 [31]. P20Bap31 propagates the apoptotic signal from ER back to mitochondria by stimulating the ER calcium release which activates and opens the mPTP and thus amplifies the death signal [58]. IP3R-Grp75-VDAC1 complex at the MAM is crucial for mitochondrial calcium uptake which could thus participate in the apoptotic signal through mitochondrial calcium overload. SUMOylation of DLP1 was also reported to stabilize the MERC and promote calcium crosstalk and cytochrome c release [59].

\section{Disturbed ER-mitochondria tethering in neurodegenerative disorders}

Alzheimer's disease (AD), Parkinson's disease (PD), and amyotrophic lateral sclerosis with associated frontotemporal dementia (ALS/FTD) are major neurodegenerative diseases. These neurodegenerative diseases are characterized by damage to various cellular processes, many of which are regulated by ER-mitochondria tethering. Indeed, abnormal MAM signaling is reported in all these neurodegenerative diseases as reviewed below.

\section{Alzheimer's Disease}

Presenilins are the catalytic subunits of the gamma secretase responsible for the generation of amyloid- $\beta$ (A $\beta$ ) [60]. Mutations in presenilin 1 (PS1) and 2 (PS2) cause human familial AD. Presenilins were present in many compartments in the cell including ER and mitochondria [61]. Eric Schon's group first demonstrated the enrichment of presenilins in the MAM and suggested that MAM is the predominant subcellular location for PS1/PS2 and gamma secretase activity and later further demonstrated significantly increased MAM function and ER-mitochondrial communication in presenilin-deficient cells and in fibroblasts from patients with both the familial and sporadic forms of $\mathrm{AD}$, suggesting that upregulated MAM function and increased ER-mitochondria crosstalk may be involved in the pathogenesis of $\mathrm{AD}[60,62,63]$. Indeed, expression of MAM-associated proteins were significantly increased in postmortem brain of human $\mathrm{AD}$ patients and in the APP transgenic mice although direct evidence of specific alterations in the ER-mitochondria interaction was lacking [64]. Consistent with this notion, $\mathrm{A} \beta$ treatment leads to increased MAM protein expression and increased MERC in cultured neurons and ApoE4 allele also upregulated MAM function [65]. However, another group, although agreeing upon the notion that presenilin modulates MAM function, disagreed on the specific effects of PS2 on MAM: Pizzo's group reported that the expression of the PS2, but not of PS1, facilitates the physical interaction and functional coupling between ER and mitochondria in Mfn2-dependent manner [66]. The reason for the discrepancy is not clear.

\section{Amyotrophic Lateral Sclerosis with associated Frontotemporal Dementia}

Mutations in genes encoding several different MAM proteins, including a tethering protein, were associated with familial ALS which provides strong evidence to support the involvement of disturbed MAM function in the pathogenesis of ALS although it is hard to reconcile whether upregulated or disrupted MAM function plays the pathogenic role. VAPB interacts with the OMM protein PTPIP51 to form a MAM tether complex [27]. P56S mutation of VAPB causes autosomal-dominant ALS and VAPB-P56S mutant demonstrated higher affinity for PTPIP51 and consequently, increased $\mathrm{Ca}^{2+}$ transfer into mitochondria [27]. On the other hand, Sig-1R is another MAM protein that participates in the IP3R function to facilitate calcium signaling at MAM [36]. Mutations in Sig-1R caused juvenile ALS although in an autosomal recessive pattern [67]. Loss of Sig-1R function by gene mutation or downregulation has been shown to break ER-mitochondria associations [68]. ALS-causing superoxide dismutase 1 (SOD1) mutants aggregated and accumulated at MAM which compromised the integrity and activity of MAM [69]. ALS/FTD-associated TAR DNA-binding protein 43 (TDP-43), and fused in sarcoma (FUS) could also disrupt the MERC and perturb mitochondrial calcium uptake from ER through activation of glycogen synthase kinase $3 \beta$ (GSK3 $\beta$ ) which in turn perturbs VAPB-PTPIP51 interaction [28].

\section{Parkinson's Disease}

Mutations in $\alpha$-synuclein gene cause autosomal dominant PD and $\alpha$-synuclein aggregates were major components of Lewy bodies. $\alpha$-synuclein is present 
in MAM [70, 71], but pathogenic point mutations of $\alpha$-synuclein result in its reduced association with MAM, coincident with a lower degree of apposition of ER with mitochondria, a decrease in MAM function, and an increase in mitochondrial fragmentation. Mutations in PARK2 gene (encoding Parkin protein) is associated with juvenile onset autosomal recessive forms of PD. Parkin is ATF4-dependently upregulated during mitochondrial and ER stress, which regulates the functional interplay between ER and mitochondria to help promote cell survival under stress [72]. Interestingly, Parkin is translocated to endoplasmic reticulum (ER) and mitochondrial/ER junctions following excitotoxicity, implicating a potential role for Parkin in MAM [73]. Indeed, a confocal microscopic study showed that Parkin overexpression resulted in enhanced physical coupling between ER and mitochondria and favored calcium transfer from the ER to the mitochondria following 1,4,5 inositol trisphosphate (InsP3) generating agonist and increased the agonist-induced ATP production in vitro [74]. Such a positive effect of Parkin on the ER-mitochondria interaction has been confirmed in nigral neurons by EM analysis [75]. However, a recent study demonstrated enhanced ER-mitochondria tethering in primary fibroblasts from Parkin knockout mice and PD patients with PARK2 gene mutations as well as in neurons derived from induced pluripotent stem cells of a patient with PARK2 gene mutations along with ER-to-mitochondria calcium transfers likely due to increased Mfn2 in MAMs [76]. PINK1, another familial PD and mitochondria quality control associated protein, and the pro-autophagic protein BECN1/Beclin1 were both found at MAM, the PINK1 and Beclin1 interaction enhanced ER-mitochondria contact and promoted the formation of autophagosome following mitophagy induction [77]. Mutations or deletion of DJ-1 are associated with autosomal recessive early onset familial PD. It was reported that DJ-1 is also present in the MAMs. DJ-1 overexpression caused increased Mitochondria-ER colocalization as demonstrated by confocal microscopy [78]. Whether this represents true increase in the MERC needs to be confirmed by other methods since confocal microscopy does not permit accurate quantification of the $<30 \mathrm{~nm}$ distance.

\section{Conclusions}

Although it remains to be fully characterized how ERmitochondria contacts are maintained and regulated, it is becoming increasingly clear that MERC provide an important platform to intertwine various signaling pathways to carry out many cellular processes of importance to neuronal function including the regulation of calcium homeostasis, phospholipid synthesis and exchange, mitochondrial biogenesis and dynamics and apoptosis. It is interesting that disturbance in MERC are involved in most of the neurodegenerative diseases studied such as $\mathrm{AD}, \mathrm{PD}$ and ALS as discussed above. Indeed, disturbance in MERC provides a connection for the various seemingly disparate features of these neurodegenerative diseases which may suggest that the disturbance in MERC may serve as a common convergent mechanism underlying neurodegeneration. Admittedly appealing, this hypothesis faces several challenges. First, the many conflicting observations, likely due to the different methods used, need to be reconciled. For example, the fundamental involvement of Mfn2 in the ER-mitochondria tethering is under hot debate and the effects of PS2 on MAM need clarification. Secondly, it appears that both upregulated and disrupted MAM functions are implicated in neurodegenerative diseases, even in the same disease. For example, VAPB-P56S mutant caused enhanced ER-mitochondria crosstalk while TDP43 and FUS disrupted ER-mitochondria contacts. While both upregulated and disrupted MAM function could lead to cellular dysfunction and neurodegeneration, this highlights the need to characterize the effects of specific neurodegenerative disease insults on the ERmitochondria axis. Thirdly, the detailed mechanism of MAM disturbance in these conditions needs to be worked out. Is there a common mechanism? TDP43 and FUS disrupt the VAPB-PTPIP51 tethering through GSK3 $\beta$ activation. While GSK3 $\beta$ is activated in many of these neurodegenerative diseases, it may not elicit the same outcome since PS1 mutation caused GSK3 $\beta$ activation [79] but led to increased ER-mitochondria tethering [80]. Fundamentally, therefore, the pathophysiological relevance of these observations also need to be firmly established.

\footnotetext{
Abbreviations

AD: Alzheimer's disease; ALS: Amyotrophic lateral sclerosis; Aß: Amyloid- $\beta$; Bap31: B-cell receptor-associated protein 31; CoA: Coenzyme A; DLP1: Dynaminlike protein; ER: Endoplasmic reticulum; FACL4: Long-chain-fatty-acid-CoA ligase 4; Fis1: Mitochondrial fission 1 protein; FTD: Frontotemporal dementia; FUS: Fused in sarcoma; grp75: Glucose-regulated protein 75; GSK3ß: Glycogen synthase kinase 33; IP3R: Inositol 1,4,5-trisphosphate receptor; MAMs: Mitochondrial associated membrane; MCU: Mitochondrial calcium uniporter; MERC: Mitochondria-ER contact/crosslink/crosstalk/communication; Mfn: Mitofusin; mPTP: Mitochondrial permeability transition pore; PACS-2: Phosphofurin acidic cluster sorting protein 2; PARK2: Parkin; PD: Parkinson's disease; PS: Presenilin; PSS: Phosphatidylserine synthase; PTPIP51: Protein tyrosine phosphatase-interacting protein-51; SERCA: Sarco-endoplasmic reticulum $\mathrm{Ca}^{2+}$ ATPase; Sig-1R: Sigma1R; SOD1: Superoxide dismutase 1; TCA: Tricarboxylic acid cycle; TDP-43: TAR DNA-binding protein 43; VAPB: Vesicle-associated membrane protein-associated protein B; VDAC: Voltage-dependent anion channel
}

\section{Acknowledgements}

Not applicable.

\section{Funding}

This work is partly supported by National Institute of Health (grant numbers NS083385 and AG049479 to XZ) and Alzheimer's Association (AARG-16-443584 to $X Z$ ). 


\section{Availability of data and materials}

Not applicable.

\section{Authors' contributions}

$Y L$ and $X Z$ conceived and wrote the manuscript. Both authors read and approved the final manuscript

\section{Ethics approval and consent to participate}

Not applicable.

\section{Consent for publication}

Not applicable.

\section{Competing interests}

X.Z. is a paid consultant of Sierra Research Group LLC.

\section{Received: 15 April 2017 Accepted: 1 August 2017}

Published online: 23 August 2017

\section{References}

1. Vance JE. Phospholipid synthesis in a membrane fraction associated with mitochondria. J Biol Chem. 1990;265(13):7248-56.

2. Montisano DF, et al. Association between mitochondria and rough endoplasmic reticulum in rat liver. Anat Rec. 1982;203(4):441-50.

3. Pickett $C B$, et al. The physical association between rat liver mitochondria and rough endoplasmic reticulum. I. Isolation, electron microscopic examination and sedimentation equilibrium centrifugation analyses of rough endoplasmic reticulum-mitochondrial complexes. Exp Cell Res. 1980;128(2):343-52.

4. Rowland AA, Voeltz GK. Endoplasmic reticulum-mitochondria contacts: function of the junction. Nat Rev Mol Cell Biol. 2012;13(10):607-25.

5. Kornmann B. The molecular hug between the ER and the mitochondria. Curr Opin Cell Biol. 2013;25(4):443-8.

6. Rizzuto $\mathrm{R}$, et al. Close contacts with the endoplasmic reticulum as determinants of mitochondrial Ca2+ responses. Science. 1998;280(5379):1763-6.

7. Csordas G, et al. Structural and functional features and significance of the physical linkage between ER and mitochondria. J Cell Biol. 2006;174(7):915-21.

8. Boehning D, et al. Cytochrome $\mathrm{c}$ binds to inositol $(1,4,5)$ trisphosphate receptors, amplifying calcium-dependent apoptosis. Nat Cell Biol. 2003;5(12):1051-61.

9. Patergnani $\mathrm{S}$, et al. Calcium signaling around mitochondria associated membranes (MAMs). Cell Commun Signal. 2011;9(1):19.

10. Hajnoczky G, Csordas G, Yi M. Old players in a new role: mitochondriaassociated membranes, VDAC, and ryanodine receptors as contributors to calcium signal propagation from endoplasmic reticulum to the mitochondria. Cell Calcium. 2002:23(5):363-77.

11. Pinton $\mathrm{P}$, et al. Calcium and apoptosis: ER-mitochondria $\mathrm{Ca} 2+$ transfer in the control of apoptosis. Oncogene. 2008;27(50):6407-18.

12. Wang $\mathrm{HJ}$, et al. Calcium regulates the association between mitochondria and a smooth subdomain of the endoplasmic reticulum. J Cell Biol. 2000;150(6):1489-98.

13. Vance JE. MAM (mitochondria-associated membranes) in mammalian cells: lipids and beyond. Biochim Biophys Acta. 2014;1841(4):595-609.

14. Friedman JR, et al. ER tubules mark sites of mitochondrial division. Science. 2011;334(6954):358-62

15. Hamasaki M, et al. Autophagosomes form at ER-mitochondria contact sites. Nature. 2013:495(7441):389-93.

16. van Vliet AR, Verfaillie T, Agostinis P. New functions of mitochondria associated membranes in cellular signaling. Biochim Biophys Acta. 2014;1843(10):2253-62

17. Cardenas $C$, et al. Essential regulation of cell bioenergetics by constitutive InsP3 receptor Ca2+ transfer to mitochondria. Cell. 2010;142(2):270-83.

18. de Brito OM, Scorrano L. Mitofusin 2 tethers endoplasmic reticulum to mitochondria. Nature. 2008:456(7222):605-10.

19. Merkwirth C, Langer T. Mitofusin 2 builds a bridge between ER and mitochondria. Cell. 2008;135(7):1165-7.

20. Daniele T, Hurbain I, Vago R, Casari G, Raposo G, Tacchetti C, Schiaffino MV Mitochondria and melanosomes establish physical contacts modulated by Mfn2 and involved in organelle biogenesis. Curr Biol. 2014;24(4):393-403.
21. Sugiura A, et al. MITOL regulates endoplasmic reticulum-mitochondria contacts via Mitofusin2. Mol Cell. 2013:51(1):20-34.

22. Naon, D., Zaninello, M., Giacomello, M., Varanita, T., Grespi, F., Lakshminaranayan, S., ... \& Zorzano, A., Critical reappraisal confirms that Mitofusin 2 is an endoplasmic reticulum-mitochondria tether. Proc Natl Acad Sci 2016. 113(40): p. 11249-11254.

23. Filadi $\mathrm{R}$, et al. Mitofusin 2 ablation increases endoplasmic reticulummitochondria coupling. Proc Natl Acad Sci U S A. 2015;112(17):E2174-81.

24. Leal NS, et al. Mitofusin-2 knockdown increases ER-mitochondria contact and decreases amyloid beta-peptide production. J Cell Mol Med. 2016:20(9):1686-95.

25. Wang PT, et al. Distinct mechanisms controlling rough and smooth endoplasmic reticulum contacts with mitochondria. J Cell Sci. 2015;128(15):2759-65.

26. Kanekura K, et al. Characterization of amyotrophic lateral sclerosis-linked P56S mutation of vesicle-associated membrane protein-associated protein B (VAPB/ALS8). J Biol Chem. 2006;281(40):30223-33.

27. De Vos KJ, et al. VAPB interacts with the mitochondrial protein PTPIP51 to regulate calcium homeostasis. Hum Mol Genet. 2012;21(6):1299-311.

28. Stoica $R$, et al. ER-mitochondria associations are regulated by the VAPB-PTPIP5 interaction and are disrupted by ALS/FTD-associated TDP-43. Nat Commun. 2014;5:3996.

29. Chandra D, et al. Association of active caspase 8 with the mitochondrial membrane during apoptosis: potential roles in cleaving BAP31 and caspase 3 and mediating mitochondrion-endoplasmic reticulum cross talk in etoposide-induced cell death. Mol Cell Biol. 2004;24(15):6592-607.

30. Breckenridge DG, et al. Caspase cleavage product of BAP31 induces mitochondrial fission through endoplasmic reticulum calcium signals, enhancing cytochrome c release to the cytosol. J Cell Biol. 2003;160(7):1115-27.

31. Iwasawa R, et al. Fis1 and Bap31 bridge the mitochondria-ER interface to establish a platform for apoptosis induction. EMBO J. 2011:30(3):556-68.

32. Simmen T, et al. PACS-2 controls endoplasmic reticulum-mitochondria communication and bid-mediated apoptosis. EMBO J. 2005:24(4):717-29.

33. Betz C, et al. mTOR complex 2-Akt signaling at mitochondria-associated endoplasmic reticulum membranes (MAM) regulates mitochondrial physiology. Proc Natl Acad Sci U S A. 2013;110(31):12526-34.

34. Szabadkai $G$, et al. Chaperone-mediated coupling of endoplasmic reticulum and mitochondrial Ca2+ channels. J Cell Biol. 2006;175(6):901-11.

35. De Stefani $D$, et al. VDAC1 selectively transfers apoptotic Ca2+ signals to mitochondria. Cell Death Differ. 2012;19(2):267-73.

36. Hayashi T, Su TP. Sigma-1 receptor chaperones at the ER-mitochondrion interface regulate $\mathrm{ca}(2+)$ signaling and cell survival. Cell. 2007;131(3):596-610.

37. Voelker DR. Bridging gaps in phospholipid transport. Trends Biochem Sci. 2005:30(7):396-404.

38. Stone SJ, Vance JE. Phosphatidylserine synthase-1 and -2 are localized to mitochondria-associated membranes. J Biol Chem. 2000:275(44):34534-40.

39. Achleitner $\mathrm{G}$, et al. Synthesis and intracellular transport of aminoglycerophospholipids in permeabilized cells of the yeast, Saccharomyces Cerevisiae. J Biol Chem. 1995;270(50):29836-42.

40. Prasad $M$, et al. Mitochondria-associated endoplasmic reticulum membrane (MAM) regulates steroidogenic activity via steroidogenic acute regulatory protein (StAR)-voltage-dependent anion channel 2 (VDAC2) interaction. J Biol Chem. 2015;290(5):2604-16.

41. Lewin TM, et al. Acyl-CoA synthetase isoforms 1, 4, and 5 are present in different subcellular membranes in rat liver and can be inhibited independently. J Biol Chem. 2001:276(27):24674-9.

42. Lewin TM, et al. Rat liver acyl-CoA synthetase 4 is a peripheral-membrane protein located in two distinct subcellular organelles, peroxisomes, and mitochondrial-associated membrane. Arch Biochem Biophys. 2002;404(2): 263-70.

43. Gellerich FN, et al. The regulation of OXPHOS by extramitochondrial calcium. Biochim Biophys Acta. 2010;1797(6-7):1018-27.

44. Baumgartner HK, et al. Calcium elevation in mitochondria is the main $\mathrm{Ca} 2+$ requirement for mitochondrial permeability transition pore (mPTP) opening. J Biol Chem. 2009:284(31):20796-803.

45. Baughman JM, et al. Integrative genomics identifies MCU as an essential component of the mitochondrial calcium uniporter. Nature. 2011;476(7360):341-5

46. Oi H, Li L, Shuai J. Optimal microdomain crosstalk between endoplasmic reticulum and mitochondria for Ca2+ oscillations. Sci Rep. 2015;5:7984.

47. Patron $\mathrm{M}$, et al. The mitochondrial calcium uniporter (MCU): molecular identity and physiological roles. J Biol Chem. 2013;288(15):10750-8. 
48. Bononi A, et al. Mitochondria-associated membranes (MAMs) as hotspot $\mathrm{ca}(2+)$ signaling units. Adv Exp Med Biol. 2012;740:411-37.

49. Horne JH, Meyer T. Elementary calcium-release units induced by inositol trisphosphate. Science. 1997:276(5319):1690-3.

50. White $\mathrm{C}$, et al. The endoplasmic reticulum gateway to apoptosis by $\mathrm{BCl}-\mathrm{X}(\mathrm{L})$ modulation of the InsP3R. Nat Cell Biol. 2005:7(10):1021-8.

51. Bonneau $\mathrm{B}$, et al. IRBIT controls apoptosis by interacting with the $\mathrm{BCl}-2$ homolog, Bcl2110, and by promoting ER-mitochondria contact. Elife. 2016;5:e19896.

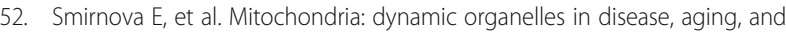
development. Cell. 2006;125(7):1241-52.

53. Smirnova E, et al. Dynamin-related protein Drp1 is required for mitochondrial division in mammalian cells. Mol Biol Cell. 2011;12(8):2245-56.

54. Yoon Y, Pitts KR, MCNiven MA. Mammalian dynamin-like protein DLP1 tubulates membranes. Mol Biol Cell. 2001;12(9):2894-905.

55. Shim SH, et al. Super-resolution fluorescence imaging of organelles in live cells with photoswitchable membrane probes. Proc Natl Acad Sci U S A. 2012;109(35):13978-83

56. Korobova F, Ramabhadran V, Higgs HN. An actin-dependent step in mitochondrial fission mediated by the ER-associated formin INF2. Science. 2013:339(6118):464-7.

57. Grimm S. The ER-mitochondria interface: the social network of cell death. Mol Cell Res. 2012;1823(2):327-34

58. Wang B, et al. Fis1, Bap31 and the kiss of death between mitochondria and endoplasmic reticulum. EMBO J. 2011;30(3):451-2.

59. Prudent J, et al. MAPL SUMOylation of Drp1 stabilizes an ER/mitochondrial platform required for cell death. Mol Cell. 2015;59(6):941-55.

60. Area-Gomez $\mathrm{E}$, et al. Presenilins are enriched in endoplasmic reticulum membranes associated with mitochondria. Am J Pathol. 2009;175(5):1810-6.

61. Zampese $E_{1}$ et al. Presenilin 2 modulates endoplasmic reticulum (ER)-mitochondria interactions and Ca2+ cross-talk. Proc Natl Acad Sci U S A. 2011;108(7):2777-82.

62. Schon EA, Area-Gomez E. Is Alzheimer's disease a disorder of mitochondriaassociated membranes? J Alzheimers Dis. 2010;20(Suppl 2):S281-92.

63. Schreiner $B$, et al. Amyloid- $\beta$ peptides are generated in mitochondria-associated endoplasmic reticulum membranes. J Alzheimers Dis. 2015:43(2):369-74.

64. Hedskog $L$, et al. Modulation of the endoplasmic reticulum-mitochondria interface in Alzheimer's disease and related models. Proc Natl Acad Sci U S A. 2013;110(19):7916-21.

65. Tambini MD, et al. ApoE4 upregulates the activity of mitochondria-associated ER membranes. EMBO Rep. 2016;17(1):27-36.

66. Filadi $\mathrm{R}$, et al. Presenilin 2 modulates endoplasmic reticulum-mitochondria coupling by tuning the antagonistic effect of Mitofusin 2. Cell Rep. 2016;15(10):2226-38

67. Al-Saif A, Al-Mohanna F, Bohlega S. A mutation in sigma-1 receptor causes juvenile amyotrophic lateral sclerosis. Ann Neurol. 2011;70(6):913-9.

68. Su TP, et al. The sigma-1 receptor chaperone as an inter-organelle signaling modulator. Trends Pharmacol Sci. 2010;31(12):557-66.

69. Watanabe $\mathrm{S}$, et al. Mitochondria-associated membrane collapse is a common pathomechanism in SIGMAR1- and SOD1-linked ALS. EMBO Mol Med. 2016;8(12):1421-37.

70. Guardia-Laguarta C, et al. Alpha-Synuclein is localized to mitochondria-associated ER membranes. J Neurosci. 2014;34(1):249-59.

71. Cali T, et al. Alpha-Synuclein controls mitochondrial calcium homeostasis by enhancing endoplasmic reticulum-mitochondria interactions. J Biol Chem 2012;287(22):17914-29.

72. Bouman $L$, et al. Parkin is transcriptionally regulated by ATF4: evidence for an interconnection between mitochondrial stress and ER stress. Cell Death Differ. 2011;18(5):769-82.

73. Van Laar VS, et al. Glutamate excitotoxicity in neurons triggers mitochondrial and endoplasmic reticulum accumulation of Parkin, and, in the presence of N-acetyl cysteine, mitophagy. Neurobiol Dis. 2015;74:180-93.

74. Cali T, et al. Enhanced parkin levels favor ER-mitochondria crosstalk and guarantee $\mathrm{ca}(2+)$ transfer to sustain cell bioenergetics. Biochim Biophys Acta. 2013;1832(4):495-508.

75. Zheng $L$, et al. Parkin functionally interacts with PGC-1alpha to preserve mitochondria and protect dopaminergic neurons. Hum Mol Genet. 2017:26(3):582-98

76. Gautier CA, et al. The endoplasmic reticulum-mitochondria interface is perturbed in PARK2 knockout mice and patients with PARK2 mutations. Hum Mol Genet. 2016;25(14):2972-84.
77. Gelmetti V, et al. PINK1 and BECN1 relocalize at mitochondria-associated membranes during mitophagy and promote ER-mitochondria tethering and autophagosome formation. Autophagy. 2017;13(4):654-69.

78. Ottolini D, et al. The Parkinson disease-related protein DJ-1 counteracts mitochondrial impairment induced by the tumour suppressor protein p53 by enhancing endoplasmic reticulum-mitochondria tethering. Hum $\mathrm{Mol}$ Genet. 2013;22(11):2152-68.

79. Pigino $\mathrm{G}$, et al. Alzheimer's presenilin 1 mutations impair kinesin-based axonal transport. J Neurosci. 2003;23(11):4499-508.

80. Area-Gomez E, et al. Upregulated function of mitochondria-associated ER membranes in Alzheimer disease. EMBO J. 2012:31(21):4106-23.

\section{Submit your next manuscript to BioMed Central and we will help you at every step:}

- We accept pre-submission inquiries

- Our selector tool helps you to find the most relevant journal

- We provide round the clock customer support

- Convenient online submission

- Thorough peer review

- Inclusion in PubMed and all major indexing services

- Maximum visibility for your research

Submit your manuscript at www.biomedcentral.com/submit
C) Biomed Central 